Discrete ANALYSis, 2018:14, 11 pp.

www.discreteanalysisjournal.com

\title{
On Isoperimetric Stability
}

\author{
Vsevolod F. Lev \\ Received 19 September 2017; Published 6 August 2018
}

\begin{abstract}
We show that a non-empty subset of an abelian group with a small edge boundary must be large; in particular, if $A$ and $S$ are finite, non-empty subsets of an abelian group such that $S$ is independent, and the edge boundary of $A$ with respect to $S$ does not exceed $(1-\gamma)|S||A|$ with a real $\gamma \in(0,1]$, then $|A| \geq 4^{(1-1 / d) \gamma|S|}$, where $d$ is the smallest order of an element of $S$. Here the constant 4 is best possible.

As a corollary, we derive an upper bound for the size of the largest independent subset of the set of popular differences of a finite subset of an abelian group. For groups of exponent 2 and 3, our bound translates into a sharp estimate for the additive dimension of the popular difference set.

We also prove, as an auxiliary result, the following estimate of possible independent interest: if $A \subseteq \mathbb{Z}^{n}$ is a finite, non-empty downset then, denoting by $w(a)$ the number of non-zero components of the vector $a \in A$, we have
\end{abstract}

$$
\frac{1}{|A|} \sum_{a \in A} w(a) \leq \frac{1}{2} \log _{2}|A|
$$

Key words and phrases: stability, edge boundary

\section{Summary of Results}

Let $G$ be an abelian group. For finite subsets $A, S \subseteq G$, denote by $\partial_{S}(A)$ the number of edges from $A$ to its complement $G \backslash A$ in the directed Cayley graph, induced on $G$ by $S$; that is,

$$
\partial_{S}(A)=|\{(a, s) \in A \times S: a+s \notin A\}| .
$$

In a trivial way, we have $0 \leq \partial_{S}(A) \leq|S||A|$. Clearly, if $\partial_{S}(A)=0$, then $A$ is a union of cosets of the subgroup $\langle S\rangle$ generated by $S$; in particular, $|A| \geq|\langle S\rangle|$. What can be said about $A$ if we are given that 
$\partial_{S}(A) \leq(1-\gamma)|S||A|$, with some $\gamma \in(0,1]$ ? How small can $|A|$ be under this assumption? In the case where $G$ is homocyclic of exponent not exceeding 4 (that is, $G=C_{m}^{n}$ with $m \in\{2,3,4\}$ and $n \geq 1$ ), and $S$ is a standard generating subset of $G$, the answer is given by the following easy consequence of [L15, Corollary 1.10].

Theorem 1. Let $G$ be a homocyclic group of exponent $\exp (G) \in\{2,3,4\}$ and $\operatorname{rank} n:=\operatorname{rk} G$. If $A \subseteq G$ is non-empty and $\partial_{S}(A) \leq(1-\gamma) n|A|$ with a generating subset $S \subseteq G$ and real $\gamma \in(0,1]$, then

$$
|A| \geq|G|^{\gamma}
$$

We postpone the proofs of Theorem 1 and other results presented in this section to Sections $2-4$.

The following examples show that the assumption of Theorem 1 cannot be relaxed to $\partial_{S}(A) \leq$ $(1-\gamma)|S||A|$, and that the conclusion $|A| \geq|G|^{\gamma}$ is nearly best possible.

Example 1. Fix $m \geq 2$ and suppose that $k, n \geq 1$ are integers such that $k=\log _{m} n+O(1)$, with an absolute implicit constant. Let $\left\{e_{1}, \ldots, e_{n}\right\}$ be a standard generating subset of the group $C_{m}^{n}$, and consider the sets $A:=\left\langle e_{1}, \ldots, e_{k}\right\rangle$ and $S:=A \cup\left\{e_{k+1}, \ldots, e_{n}\right\}$. (We keep using the standard notation $\langle T\rangle$ for the subgroup of an abelian group generated by its subset $T$.) We have then $|S|=m^{k}+n-k$ and $\partial_{S}(A)=(n-k)|A|=(1-\gamma)|S||A|$ where, writing $\tau:=m^{k} / n$,

$$
\gamma=\frac{m^{k}}{m^{k}+n-k} \approx \frac{\tau}{\tau+1}
$$

At the same time, $|A|=m^{k}$ is much smaller than $\left|C_{m}^{n}\right|^{\gamma}=m^{\gamma n}$.

Example 2. Suppose that $m \geq 2$ and $k, n \geq 1$ are integers with $k \mid n$. Let $C_{m}^{n}=H_{1} \oplus \cdots \oplus H_{k}$ be a direct sum decomposition with every subgroup $H_{i}$ isomorphic to $C_{m}^{n / k}$, and let $S \subseteq C_{m}^{n}$ be an $n$-element generating subset having exactly $n / k$ elements in every subgroup $H_{i}$. Consider the set $A:=H_{1} \cup \cdots \cup H_{k}$. It is easily seen that $|A|=\left(m^{n / k}-1\right) k+1$ and $\partial_{S}(A)=\left(m^{n / k}-1\right)(k-1) n$. Consequently, letting $\gamma:=k^{-1}$, we have $\partial_{S}(A)<(1-\gamma) n|A|$, while $|A| \leq m^{n / k} k=\gamma^{-1}\left|C_{m}^{n}\right|^{\gamma}$.

In the case where $\exp (G) \in\{2,3\}$, Theorem 1 is easy to extend to the situation where $S$ is an arbitrary (not necessarily generating) subset of $G$.

Corollary 1. Suppose that $A$ and $S$ are finite, non-empty subsets of an abelian group $G$ of exponent $\exp (G) \in\{2,3\}$. Let $H:=\langle S\rangle$ and write $n:=\operatorname{rk} H$. If $\partial_{S}(A) \leq(1-\gamma) n|A|$ with a real $\gamma \in(0,1]$, then

$$
|A| \geq|H|^{\gamma}
$$

Interestingly, Theorem 1 does not allow a straightforward extension to the case where $\exp (G)>4$; this is demonstrated by our next example.

Example 3. Suppose that $1<t<m$ and $n \geq 1$ are integers, and consider the set $A:=[0, t-1]^{n} \subseteq C_{m}^{n}$; thus, $|A|=t^{n}$, and it is easily seen that if $S$ is a standard generating set in $C_{m}^{n}$, then $\partial_{S}(A)=n t^{n-1}$. Consequently, letting $\gamma:=1-t^{-1}$, we have $\partial_{S}(A)=(1-\gamma) n|A|$ while $|A|=b^{\gamma_{n}}$, where $b=t^{\gamma^{-1}}=\exp (t \log t /(t-1))$ can be as small as 4 (attained for $t=2$ ). Therefore, instead of the estimate $|A| \geq|G|^{\gamma}$ of Theorem 1 , the best estimate one can hope for in the general case is $|A| \geq 4^{\gamma n}$, where $n=\operatorname{rk} G$. 
We say that a finite subset $S$ of an abelian group is independent if for any integer-valued function $k$ on $S$, we have $\sum_{s \in S} k(s) s \neq 0$, unless all summands are equal to 0 ; that is, the sum $\oplus_{s \in S}\langle s\rangle$ is direct.

Extending Theorem 1 to arbitrary abelian groups, we have the following result.

Theorem 2. Suppose that $A$ and $S$ are finite, non-empty subsets of an abelian group such that $S$ is independent. Write $n:=|S|$ and $d:=\min \{\operatorname{ord} s: s \in S\}$. If $\partial_{S}(A) \leq(1-\gamma) n|A|$ with a real $\gamma \in(0,1]$, then

$$
|A| \geq 4^{(1-1 / d) \gamma n} \text {. }
$$

The statement of Theorem 2 is to be interpreted the expected way if some, or all, of the orders of the elements of $S$ are infinite; in particular, in the latter case the conclusion of the theorem should be read as $|A| \geq 4^{\gamma n}$.

As Example 2 shows, the coefficient $1-1 / d$ in the exponent is best possible for $d=2$, and cannot be replaced with a number larger than $\log 3 / \log 4 \approx 0.792$ for $d=3$.

Let $\mathbb{Z}_{\geq 0}$ denote the set of non-negative integers. A set $A \subseteq \mathbb{Z}_{\geq 0}^{n}$ is called a downset if for every $a \in A$ and every $z \in \mathbb{Z}_{\geq 0}^{n}$ majorated by $a$ coordinate-wise, we have $z \in A$. The weight of a vector $z \in \mathbb{Z}^{n}$, denoted below $w(z)$, is the number of non-zero coordinates of $z$.

The following estimate for the average weight of a vector in a downset in $\mathbb{Z}_{\geq 0}^{n}$ is an important ingredient of the proof of Theorem 2 and, we believe, may be found interesting in its own right.

Theorem 3. If $n \geq 1$ is an integer and $A \subseteq \mathbb{Z}_{\geq 0}^{n}$ is a finite, non-empty downset, then

$$
\frac{1}{|A|} \sum_{a \in A} w(a) \leq \frac{1}{2} \log _{2}|A| .
$$

It is easy to see that for sets of the form $A=\left[0, l_{1}\right] \times \cdots \times\left[0, l_{n}\right]$ with $l_{1}, \ldots, l_{n} \in\{0,1\}$, equality is attained in the estimate of Theorem 3.

Theorem 3 looks strikingly similar to [R03, Theorem 1.1] which says that if a set $A \subseteq\{0,1\}^{n}$ is union-closed (that is, $a_{1}, a_{2} \in A$ implies $a_{1} \vee a_{2} \in A$ ), then $\frac{1}{|A|} \sum_{a \in A} w(a) \geq \frac{1}{2} \log _{2}|A|$. However, it seems that the two results cannot be reduced to each other.

For a somewhat unexpected link between (the proof of) Theorem 3 and the Loomis-Whitney inequality, see a remark in Section 5.

In Section 3 we extend Theorem 3 to arbitrary abelian groups.

One can equivalently restate Theorem 3 in terms of multisets. Formally, a (finite) multiset with the ground set $S$ is a finitely supported function from $S$ to $\mathbb{Z}_{\geq 0}$; the value of the function at the element $s \in S$ is the multiplicity of $s$ in the multiset. The cardinality of the multiset $A$ is the $\operatorname{sum} \sum_{s \in \operatorname{supp} A} A(s)$. For a multiset $A$ and an element $s \in S$, we write $s \in A$ if $A(s)>0$, and we define the multiset $A \backslash\{s\}$ by

$$
A \backslash\{s\}: t \mapsto \begin{cases}A(t), & t \in S, t \neq s, \\ \max \{A(s)-1,0\}, & t=s .\end{cases}
$$

The family $\mathcal{A}$ of multisets (sharing the same ground set $S$ ) is monotonic if $A \in \mathcal{A}$ implies $A \backslash\{s\} \in \mathcal{A}$ for each $s \in S$.

Since every element of $\mathbb{Z}_{\geq 0}^{n}$ defines a multiset with the ground set $[n]$, and a set in $\mathbb{Z}_{\geq 0}^{n}$ is a downset if and only if the corresponding family of multisets is monotonic, we have the following restatement of Theorem 3. 
Theorem $\mathbf{3}^{\prime}$. If $\mathcal{A}$ is a finite, non-empty, monotonic family of multisets, then

$$
\frac{1}{|\mathcal{A}|} \sum_{A \in \mathcal{A}}|\operatorname{supp} A| \leq \frac{1}{2} \log _{2}|\mathcal{A}| .
$$

For a finite subset $A$ of an abelian group $G$, denote by $\operatorname{dim}_{I}(A)$ the largest size of an independent subset of $A$. Given an element $g \in G$, define $r_{A}(g)$ to be the number of representations of $g$ as a difference of two elements of $A$, and for real $\gamma \in(0,1]$ let $P_{\gamma}(A)$ be the set of all $\gamma$-popular differences in $A$; that is,

$$
r_{A}(g):=\left|\left\{\left(a, a^{\prime}\right) \in A \times A: g=a-a^{\prime}\right\}\right|
$$

and

$$
P_{\gamma}(A):=\left\{g \in G: r_{A}(g) \geq \gamma|A|\right\} .
$$

As a simple corollary of Theorems 1 and 2, in Section 4 we prove

Theorem 4. If $p$ is the smallest order of a non-zero element of an abelian group $G$, then for any finite, non-empty subset $A \subseteq G$ and real $\gamma \in[0,1)$, we have

$$
\operatorname{dim}_{I}\left(P_{\gamma}(A)\right) \leq(2(1-1 / p))^{-1} \gamma^{-1} \log _{2}|A| .
$$

Moreover, if $\exp (G)=3$, then indeed we have

$$
\operatorname{dim}_{I}\left(P_{\gamma}(A)\right) \leq \gamma^{-1} \log _{3}|A| .
$$

In the situation where $G$ is homocyclic of exponent $m$, the estimate of Theorem 4 is sharp for $m \in\{2,3\}$, and reasonably close to sharp for $m \geq 4$. To see this we essentially return back to Example 2 to review it from a slightly different perspective.

Example 4. Fix integers $m \geq 2$ and $k, n \geq 1$ with $k \mid n$, and consider a direct sum decomposition $C_{m}^{n}=$ $H_{1} \oplus \cdots \oplus H_{k}$ where each of $H_{1}, \ldots, H_{k}<C_{m}^{n}$ is isomorphic to $C_{m}^{n / k}$. Let $A:=H_{1} \cup \cdots \cup H_{k}$, so that $|A|=k\left(m^{n / k}-1\right)+1 \leq k m^{n / k}$ and every non-zero element $a \in A$ satisfies $r_{A}(a)=m^{n / k}$. Setting $\gamma:=$ $m^{n / k} /|A| \geq k^{-1}$, we then have

$$
\operatorname{dim}_{I}\left(P_{\gamma}(A)\right) \geq n=k \log _{m}(\gamma|A|) \geq \gamma^{-1} \log _{m}|A|-\gamma^{-1} \log _{m}\left(\gamma^{-1}\right)
$$

It is interesting to compare Theorem 4 with a result of Shkredov and Yekhanin [SY11, Theorem 3.1]. To this end we recall that a subset $A$ of an abelian group is called dissociated if the subset sums $\sum_{a \in B} a$ are pairwise distinct, for all subsets $B \subseteq A$. The additive dimension of $A$, which we denote $\operatorname{dim}_{D}(A)$, is the size of the largest dissociated subset of $A$. The result of Shkredov-Yekhanin essentially says that if $A$ is a subset of a finite abelian group, then

$$
\operatorname{dim}_{D}\left(P_{\gamma}(A)\right) \ll \gamma^{-1} \log |A|
$$

with an absolute implicit constant. It is readily seen that every independent set in an abelian group is dissociated, and that for the groups of exponent 2 and 3, the two notions coincide. As a result, we have $\operatorname{dim}_{I}(P) \leq \operatorname{dim}_{D}(P)$, for every subset $P$ of the group, with equality for groups of exponent 2 or 3 . 
Consequently, the Shkredov-Yekhanin bound (1) is qualitatively stronger than Theorem 4 for groups of exponent larger than 3, while Theorem 4 is stronger than (1) for groups of exponent 2 and 3 (providing the sharp coefficients in this case).

We now turn to the proofs of the results discussed above; Theorem 3 will be proved in the next section, Theorems 1 and 2 and Corollary 1 in Section 3, and Theorem 4 in Section 4. Concluding remarks and open problems are gathered in Section 5.

\section{Proof of Theorem 3}

Given an integer $n \geq 1$, let $\left\{e_{1}, \ldots, e_{n}\right\}$ be the standard basis of $\mathbb{R}^{n}$, and for each $i \in[1, n]$ denote by $\mathcal{L}_{i}$ the $i$ th coordinate hyperplane, and by $\pi_{i}$ the orthogonal projection of $\mathbb{R}^{n}$ onto $\mathcal{L}_{i}$.

We use induction by $n$, and for every fixed value of $n$ by $|A|$. If $n=1$, then

$$
\frac{1}{|A|} \sum_{a \in A} w(a)=1-\frac{1}{|A|} \leq \frac{1}{2} \log _{2}|A|
$$

as one can easily verify. Also, the estimate in question is immediate if $|A|=1$. Suppose therefore that $n \geq 2$ and also $|A| \geq 2$.

Since the set $A$ is a downset, it has exactly $\left|\pi_{i}(A)\right|$ elements on the $i$ th coordinate hyperplane $\mathcal{L}_{i}$, for every $i \in[1, n]$. Consequently, double-counting gives

$$
\begin{aligned}
\sum_{a \in A} w(a) & =\sum_{i=1}^{n}\left|\left\{a \in A: e_{i} \in \operatorname{supp} a\right\}\right| \\
& =\sum_{i=1}^{n}\left(|A|-\left|\pi_{i}(A)\right|\right) \\
& =n|A|-\left(\left|\pi_{1}(A)\right|+\cdots+\left|\pi_{n}(A)\right|\right),
\end{aligned}
$$

and we thus want to prove that

$$
n|A| \leq\left|\pi_{1}(A)\right|+\cdots+\left|\pi_{n}(A)\right|+\frac{1}{2}|A| \log _{2}|A| .
$$

Using again the assumption that $A$ is a downset, we conclude that its projection onto the $n$th coordinate axis is an interval $[0, l]$ with an integer $l \geq 0$, and we partition $A$ as $A=B \cup\left(l e_{n}+C\right)$ where $C \subseteq \mathcal{L}_{n}$ and $B \cap\left(l e_{n}+\mathcal{L}_{n}\right)=\varnothing$.

If $B=\varnothing$, then $A \subseteq \mathcal{L}_{n}$; consequently,

$$
(n-1)|A| \leq\left|\pi_{1}(A)\right|+\cdots+\left|\pi_{n-1}(A)\right|+\frac{1}{2}|A| \log _{2}|A|
$$

by the induction hypothesis, and combining this with $\left|\pi_{n}(A)\right|=|A|$ we get the assertion.

If $B \neq \varnothing$, then both $B \subseteq \mathbb{Z}_{\geq 0}^{n}$ and $C \subseteq \mathbb{Z}_{\geq 0}^{n-1}$ are downsets, and the induction hypothesis gives

$$
(n-1)|C| \leq\left|\pi_{1}(C)\right|+\cdots+\left|\pi_{n-1}(C)\right|+\frac{1}{2}|C| \log _{2}|C|
$$


and

$$
n|B| \leq\left|\pi_{1}(B)\right|+\cdots+\left|\pi_{n}(B)\right|+\frac{1}{2}|B| \log _{2}|B| .
$$

Since $\left|\pi_{i}(A)\right|=\left|\pi_{i}(B)\right|+\left|\pi_{i}(C)\right|$ for $i \in[1, n-1]$, and $\pi_{n}(A)=\pi_{n}(B)$ by the downset assumption, to complete the proof it suffices to show that

$$
|C|+\frac{1}{2}|C| \log _{2}|C|+\frac{1}{2}|B| \log _{2}|B| \leq \frac{1}{2}|A| \log _{2}|A| ;
$$

the assertion will then follow by adding together (2), (3), and (4). To prove (4) we let $\tau:=|B| /|C|$, so that $\tau \geq 1$. Dividing through by $|C|$ and substituting $|B|=\tau|C|$ and $|A|=(\tau+1)|C|$ into (4) brings it to the form

$$
1+\frac{1}{2} \tau \log _{2} \tau \leq \frac{1}{2}(\tau+1) \log _{2}(\tau+1),
$$

an inequality which is easy to verify using basic calculus.

\section{Proofs of Corollary 1 and Theorems 1 and 2}

As indicated above, Theorem 1 is an immediate consequence of [L15, Corollary 1.10], which says that if $G$ is a finite abelian group of exponent $m \in\{2,3,4\}$, then for any generating subset $S \subseteq G$ and any non-empty subset $A \subseteq G$ one has $\partial_{S}(A) \geq|A| \log _{m}(|G| /|A|)$; combined with the assumption $\partial_{S}(A) \leq(1-\gamma) n|A|$, this yields $\log _{m}(|G| /|A|) \leq(1-\gamma) n$, and the assertion of Theorem 1 follows.

Proof of Corollary 1. Consider the coset decomposition $A=\left(a_{1}+A_{1}\right) \cup \cdots \cup\left(a_{k}+A_{k}\right)$, where $A_{1}, \ldots, A_{k} \subseteq$ $H$, and $a_{1}, \ldots, a_{k}$ represent pairwise distinct $H$-cosets. The case where $S=\{0\}$ is immediate, and we thus assume that $S \neq\{0\}$, and therefore $H$ is non-trivial. From

$$
\sum_{i=1}^{k} \partial_{S}\left(A_{i}\right)=\partial_{S}(A) \leq(1-\gamma) n|A|=(1-\gamma) n \sum_{i=1}^{k}\left|A_{i}\right|
$$

we conclude that there exists $i \in[1, k]$ with $\partial_{S}\left(A_{i}\right) \leq(1-\gamma) n\left|A_{i}\right|$. Since $\exp (G) \in\{2,3\}$ implies that also $\exp (H) \in\{2,3\}$, and therefore $H$ is homocyclic, by Theorem 1 we now get

$$
|A| \geq\left|A_{i}\right| \geq|H|^{\gamma}
$$

as required.

Below in this section we prove Theorem 2. The proof uses the compression technique in the general settings of arbitrary finite abelian groups, so we start with setting up the machinery of this general compression.

Suppose that $G$ is an abelian group, and $S=\left\{s_{1}, \ldots, s_{n}\right\} \subseteq G$ is a finite, independent, generating subset; that is, for each $i \in[1, n]$, letting $S_{i}:=S \backslash\left\{s_{i}\right\}$, we have $G=\left\langle S_{i}\right\rangle \oplus\left\langle s_{i}\right\rangle$. For integer $k \geq 0$ and elements $g, v \in G$ with ord $v \geq k$, write $P(g, v, k):=\{g, g+v, \ldots, g+(k-1) v\}$; thus, $P(g, v, k)$ is the 


\section{ON ISOPERIMETRIC STABILITY}

$k$-term arithmetic progression with the first term $g$ and difference $v$. Given a finite subset $A \subseteq G$ and an index $i \in[1, n]$, define the compression of $A$ along $s_{i}$ to be the set

$$
[A]_{i}:=\bigcup_{g \in\left\langle S_{i}\right\rangle} P\left(g, s_{i},\left|A \cap\left(g+\left\langle s_{i}\right\rangle\right)\right|\right)
$$

that is, in each $\left\langle s_{i}\right\rangle$-coset the set $[A]_{i}$ has exactly as many elements as the original set $A$, but the elements are "stacked towards the beginning of the coset". Thus, writing for every $i \in[1, n]$

$$
K_{i}:= \begin{cases}{\left[0, \operatorname{ord}\left(s_{i}\right)\right)} & \text { if } s_{i} \text { has finite order } \\ \mathbb{Z} & \text { otherwise }\end{cases}
$$

for an element $g \in G$ to lie it $[A]_{i}$, it is necessary and sufficient that in the (unique) representation $g=h+k s_{i}$ with $h \in\left\langle S_{i}\right\rangle$ and $k \in K_{i}$, we had in fact $0 \leq k<\left|\left(g+\left\langle s_{i}\right\rangle\right) \cap A\right|$.

We say that $A$ is $i$-compressed if $[A]_{i}=A$; that is, for each $g \in\left\langle S_{i}\right\rangle$ and $k \in K_{i}$, we have $g+k s_{i} \in A$ if and only if $0 \leq k<\left|\left(g+\left\langle s_{i}\right\rangle\right) \cap A\right|$. Equivalently, $A$ is $i$-compressed if $A \backslash\left\langle S_{i}\right\rangle \subseteq A+s_{i}$. Clearly, $[A]_{i}$ is $i$-compressed for any $A \subseteq G$ and $i \in[1, n]$.

The set $A \subseteq G$ is compressed with respect to $S$ if it is $i$-compressed for each $i \in[1, n]$.

We now show that compression along any element of $S$ does not destroy the property of being compressed along other elements of $S$.

Claim 1. If, for some $i, j \in[1, n]$, the set $A \subseteq G$ is $i$-compressed, then so is the set $[A]_{j}$.

Proof. The assertion is immediate if $i=j$, and we thus assume that $i \neq j$. We further assume that $A$ is $i$-compressed and show that then also $[A]_{j}$ is $i$-compressed.

It suffices to show that for any $g \in[A]_{j} \backslash\left\langle S_{i}\right\rangle$, we have $g \in[A]_{j}+s_{i}$. Since $A$ is $i$-compressed, for any $z \in\left\langle s_{j}\right\rangle$ with $g+z \in A$ we also have $g-s_{i}+z \in A$; consequently,

$$
\left|\left(g+\left\langle s_{j}\right\rangle\right) \cap A\right| \leq\left|\left(g-s_{i}+\left\langle s_{j}\right\rangle\right) \cap A\right|,
$$

whence

$$
\left|\left(g+\left\langle s_{j}\right\rangle\right) \cap[A]_{j}\right| \leq\left|\left(g-s_{i}+\left\langle s_{j}\right\rangle\right) \cap[A]_{j}\right| .
$$

Write $g=h+k s_{j}$ with $h \in\left\langle S_{j}\right\rangle$ and $k \in K_{j}$. From $g \in[A]_{j}$ we have $0 \leq k<\left|\left(g+\left\langle s_{j}\right\rangle\right) \cap[A]_{j}\right|$. Now (5) yields $0 \leq k<\left|\left(g-s_{i}+\left\langle s_{j}\right\rangle\right) \cap[A]_{j}\right|=\left|\left(h-s_{i}+\left\langle s_{j}\right\rangle\right) \cap[A]_{j}\right|$, and it follows that $g-s_{i}=\left(h-s_{i}\right)+k s_{j} \in$ $[A]_{j}$, as wanted.

Claim 2. For any $G, A$, and $S$ as above, and any $i, j \in[1, n]$, we have

$$
\left|\left\{a \in[A]_{i}: a+s_{j} \notin[A]_{i}\right\}\right| \leq\left|\left\{a \in A: a+s_{j} \notin A\right\}\right| .
$$

Consequently,

$$
\partial_{S}\left([A]_{i}\right) \leq \partial_{S}(A)
$$


Proof. The assertion follows by decomposing

$$
\left|\left\{a \in A: a+s_{j} \notin A\right\}\right|=\sum_{g \in\left\langle S_{i}\right\rangle}\left|\left\{a \in\left(g+\left\langle s_{i}\right\rangle\right) \cap A: a+s_{j} \notin A\right\}\right|
$$

and

$$
\left|\left\{a \in[A]_{i}: a+s_{j} \notin[A]_{i}\right\}\right|=\sum_{g \in\left\langle S_{i}\right\rangle}\left|\left\{a \in\left(g+\left\langle s_{i}\right\rangle\right) \cap[A]_{i}: a+s_{j} \notin[A]_{i}\right\}\right|,
$$

and observing that, for any fixed $g \in\left\langle S_{i}\right\rangle$, one has

$$
\left|\left\{a \in\left(g+\left\langle s_{i}\right\rangle\right) \cap A: a+s_{j} \notin A\right\}\right| \geq \max \left\{\left|\left(g+\left\langle s_{i}\right\rangle\right) \cap A\right|-\left|\left(g+s_{j}+\left\langle s_{i}\right\rangle\right) \cap A\right|, 0\right\},
$$

and that equality holds if $A$ gets replaced with $[A]_{i}$ :

$$
\left|\left\{a \in\left(g+\left\langle s_{i}\right\rangle\right) \cap[A]_{i}: a+s_{j} \notin[A]_{i}\right\}\right|=\max \left\{\left|\left(g+\left\langle s_{i}\right\rangle\right) \cap[A]_{i}\right|-\left|\left(g+s_{j}+\left\langle s_{i}\right\rangle\right) \cap[A]_{i}\right|, 0\right\}
$$

(recall that both intersections $\left(g+\left\langle s_{i}\right\rangle\right) \cap[A]_{i}$ and $\left(g+s_{j}+\left\langle s_{i}\right\rangle\right) \cap[A]_{i}$ are initial segments of the corresponding cosets $g+\left\langle s_{i}\right\rangle$ and $g+s_{j}+\left\langle s_{i}\right\rangle$.)

Claims 1 and 2 show that if, starting with $A$, one subsequently applies compressions along each of the elements $s_{1}, \ldots, s_{n}$, then the resulting set $\tilde{A}$ is compressed with respect to $S$, and we have $|\tilde{A}|=|A|$ and $\partial_{S}(\tilde{A}) \leq \partial_{S}(A)$.

We need the following corollary (indeed, a generalization) of Theorem 3.

Corollary 2. Suppose that $S$ is a finite, independent generating set in an abelian group G. If $A \subseteq G$ is finite, non-empty, and compressed with respect to $S$, then

$$
\frac{1}{|A|} \sum_{a \in A} w(a) \leq \frac{1}{2} \log _{2}|A|,
$$

where $w(a)$ is the number of non-zero summands in the representation of a as a linear combination of the elements from $S$.

Proof. Let $n:=|S|$, write $S=\left\{s_{1}, \ldots, s_{n}\right\}$, and consider the injective mapping $\varphi: G \rightarrow \mathbb{Z}^{n}$ defined by

$$
\varphi\left(z_{1} s_{1}+\cdots+z_{n} s_{n}\right)=\left(z_{1}, \ldots, z_{n}\right), z_{i} \in K_{i}(1 \leq i \leq n) .
$$

Clearly, the weight function on $G$ (with respect to $S$ ) agrees with that on $\varphi(G)$ (with respect to the standard basis of $\mathbb{R}^{n}$ ), and the assumption that $A$ is compressed with respect to $S$ ensures that the image $\varphi(A)$ is a downset. The assertion now follows by applying Theorem 3 to $\varphi(A)$.

Proof of Theorem 2. Let $G$ denote the underlying abelian group. We can assume that $S$ generates $G$; once the assertion is established in this case, the general case follows easily by considering the coset decomposition of $A$ as in Corollary 1.

In view of the remark following Claim 2, we can also assume without loss of generality that $A$ is compressed with respect to $S$. 


\section{ON ISOPERIMETRIC STABILITY}

Finally, we assume that at least one element of $S$ has finite order; the modifications to be made in the argument below in the case where all elements of $S$ have infinite order are straightforward (and indeed, the proof gets only simpler in this case).

Write $S=\left\{s_{1}, \ldots, s_{n}\right\}$, and for every $i \in[1, n]$, let

$$
\partial_{S}^{(i)}(A):=\left|\left\{a \in A: a+s_{i} \notin A\right\}\right|
$$

Define $N_{i}$ to be the number of $\left\langle s_{i}\right\rangle$-cosets having a non-empty intersection with $A$, and let $N_{i}^{\prime}$ be the number of $\left\langle s_{i}\right\rangle$-cosets entirely contained in $A$; thus, $N_{i}^{\prime}=0$ if the order of $s_{i}$ is infinite. We have

$$
\partial_{S}(A)=\partial_{S}^{(1)}(A)+\cdots+\partial_{S}^{(n)}(A)
$$

and, since $A$ is compressed,

$$
\partial_{S}^{(i)}(A)=N_{i}-N_{i}^{\prime}, \quad i \in[1, n] .
$$

If $s_{i}$ is of finite order then, writing $d_{i}:=\operatorname{ord} s_{i}$, in a trivial way we have

$$
\partial_{S}^{(i)}(A) \leq|A|-d_{i} N_{i}^{\prime} \leq|A|-d N_{i}^{\prime}
$$

and the resulting estimate remains true if $s_{i}$ is of infinite order. From (7) and (8),

$$
(d-1) \partial_{S}^{(i)}(A) \geq d N_{i}-|A|
$$

and then (6) along with the assumption $\partial_{S}(A) \leq(1-\gamma) n|A|$ yield

$$
(d-1)(1-\gamma) n|A| \geq(d-1) \partial_{S}(A) \geq d \sum_{i=1}^{n} N_{i}-n|A| .
$$

To address the sum in the right-hand side we notice that $N_{i}=\left|\left\langle S_{i}\right\rangle \cap A\right|=|A|-\left|A \backslash\left\langle S_{i}\right\rangle\right|$ for each $i \in[1, n]$ by the compression assumption. Consequently, in view of

$$
w(g):=\left|\left\{i \in[1, n]: g \notin\left\langle S_{i}\right\rangle\right\}\right|, \quad g \in G,
$$

we have

$$
\sum_{i=1}^{n} N_{i}=n|A|-\sum_{a \in A} w(a)
$$

Substituting into (9) and simplifying we get

$$
\frac{1}{|A|} \sum_{a \in A} w(a) \geq(1-1 / d) \gamma n
$$

and the result is now immediate from Corollary 2. 


\section{Proof of Theorem 4}

Let $S$ be an independent subset of the popular difference set $P_{\gamma}(A)$, and write $n:=|S|$. Every element $s \in S$ has at least $\gamma|A|$ representations as $s=a^{\prime}-a$ with $a, a^{\prime} \in A$. This results in at least $\gamma|A| \cdot|S|$ pairs $(a, s) \in A \times S$ with $a+s \in A$. Hence,

$$
\partial_{S}(A) \leq|A||S|-\gamma|A||S|=(1-\gamma) n|A|
$$

Consequently,

$$
|A| \geq 4^{(1-1 / p) \gamma n}
$$

by Theorem 2, which yields $n \leq(2(1-1 / p))^{-1} \gamma^{-1} \log _{2}|A|$. The first estimate follows by choosing $S$ so as to have $n=\operatorname{dim}_{I}\left(P_{\gamma}(A)\right)$.

The second estimate is obtained by replacing Theorem 2 with Corollary 1 to get

$$
|A| \geq|G|^{\gamma} \geq 3^{\gamma n}
$$

\section{Concluding Remarks and Open Problems}

As observed by Thomas Bloom (personal communication), the assumption $\partial_{S}(A) \leq(1-\gamma)|A||S|$ can be equivalently written as $\left\langle 1_{A} \circ 1_{A}, 1_{S}\right\rangle \geq \gamma|A||S|$; here $1_{A}$ and $1_{S}$ are the indicator functions of $A$ and $S$, respectively, the angle brackets are used for the scalar product of complex-valued functions on the underlying group $G$, and the "skew convolution" $f \circ g$ of the functions $f$ and $g$ is defined by $(f \circ g)(z)=\sum_{x \in G} f(x) g(x+z)$. One can use Hölder's inequality and basic Fourier analysis to conclude that if $S$ is dissociated, then $\partial_{S}(A) \leq(1-\gamma)|A||S|$ implies $|A|>\exp \left(c \gamma^{2}|S|\right)$, with an absolute constant $c>0$. This conclusion is weaker than that given by Theorem 2 , but it requires dissociativity only (instead of the more restrictive independence).

Our principal results, Theorems 1 and 2, show that a subset of an abelian group with small edge boundary must be large. It would be very interesting to understand exactly why this happens. What can be said about the structure of a set with small edge boundary?

In connection with Theorem 2, it is interesting to determine whether the following is true: for any generating subset $S$ of a finite abelian group $G$, if $\partial_{S}(A) \leq(1-\gamma) n|A|$ with $n:=\operatorname{rk} G$ and real $\gamma \in(0,1]$, then $|A| \geq 4^{(1-1 / d) \gamma n}$ (where $d$ is the minimal order of an element of $S$ ). It would also be interesting to find out whether the coefficient $1-1 / d$ in the exponent can be improved, or dropped altogether, in the case where $G$ is homocyclic of $\operatorname{exponent} \exp (G) \geq 5$ (the case $\exp (G) \leq 4$ is settled by Theorem 1 and Example 2).

In the course of the proof of Theorem 3, we have shown that any finite, non-empty downset $A \subseteq \mathbb{Z}_{\geq 0}^{n}$ satisfies

$$
n|A| \leq\left|\pi_{1}(A)\right|+\cdots+\left|\pi_{n}(A)\right|+\frac{1}{2}|A| \log _{2}|A|,
$$

where $\pi_{1}, \ldots, \pi_{n}$ are projections onto the coordinate hyperplanes. In fact, since compression can only reduce the sizes of the projections $\pi_{i}(A)$, inequality (10) holds true for any finite, non-empty set $A \subseteq \mathbb{Z}^{n}$, not necessarily a downset. Interestingly, this inequality does not follow from the famous Loomis-Whitney inequality $\left|\pi_{1}(A)\right| \cdots\left|\pi_{n}(A)\right| \geq|A|^{n-1}$ : for instance, the latter does not exclude the possibility that there is 
a set $A \subseteq \mathbb{Z}^{3}$ with $|A|=5$ and $\left|\pi_{1}(A)\right|=\left|\pi_{2}(A)\right|=\left|\pi_{3}(A)\right|=3$, while (10) shows that such a set cannot exist.

For Theorem 4, it would be interesting to determine the best possible coefficient for homocyclic groups of exponent larger than 3.

\section{Acknowledgments}

We are grateful to Thomas Bloom for the observation at the beginning of Section 5 .

\section{References}

[L15] V. LEV, Edge-isoperimetric problem for Cayley graphs and generalized Takagi function, SIAM Journal of Discrete Mathematics 29 (2015), no. 4, 2389-2411. 2, 6

[R03] D. Reimer, An average set size theorem, Combinatorics, Probability and Computing 12 (1) (2003), 89-93. 3

[SY11] I. ShKREDOV and S. YeKhAnIN, Sets with large additive energy and symmetric sets, Journal of Combinatorial Theory, Ser. A 118 (2011), no. 3, 1086-1093. 4

\section{AUTHOR}

Vsevolod F. Lev

Department of Mathematics

The University of Haifa at Oranim

Tivon 36006

Israel

seva@math.haifa.ac.il 